\title{
Spontaneous chiral symmetry breaking in liquid crystals
}

\author{
E.I. Kats \\ Landau Institute for Theoretical Physics, RAS, 2 Kosygina Str., Moscow 119334, Russia \\ E-mail: efim.i.kats@gmail.com
}

Received June 14, 2016, published online November 25, 2016

\begin{abstract}
Motivated by new experimental observations we generalize the Landau-like approach to include the direct phase transition between isotropic liquid $(I)$ and heliconical nematic liquid crystal $\left(N_{T B}\right)$ structure. We show that depending on the Landau expansion coefficients, our model allows either direct $I-N_{T B}$ transition, or the sequence of the phases $I-N-N_{T B}$ with the classical nematic liquid crystal $(N)$ sandwiched between the isotropic liquid and heliconical nematic liquid crystal. Which of these two situations is realized depends on how strong is the first order phase transition from the isotropic liquid. If it is strong enough the system undergoes $I-N-N_{T B}$ sequence, and for the very weak first order phase transition $I-N_{T B}$ transformation occurs. Furthermore in the latter case the $N_{T B}$ structure can be biaxial heliconical nematic liquid crystal.

PACS: $61.30 . \mathrm{Cz}$ Molecular and microscopic models and theories of liquid crystal structure;

61.30.Gd Orientational order of liquid crystals; electric and magnetic field effects on order;

84.37.+q Measurements in electric variables (including voltage, current, resistance, capacitance, inductance, impedance, and admittance, etc.).
\end{abstract}

Keywords: phase transitions, Landau expansion.

\section{Introduction}

Liquid crystals are fluid-like systems made of anisotropic shape molecules. As the temperature is decreased the liquid crystals undergo a series of phase transitions where the symmetry of the state is spontaneously broken. The standard, described in all textbooks (see e.g., [1-6]), road starts from a high temperature isotropic fluid, and transforms first into a nematic state, where orientational full three-dimensional rotational symmetry group $O(3)$ is broken, and then to a smectic phase, where positional (translational) order is broken in one direction. It is worth to note to this point, that long ago Ya.B. Zeldovich [7] proposed a theoretical model of "the minimal" liquid crystal with the minimal symmetry breaking (only space inversion, i.e., chiral symmetry is broken). Unfortunately for the apparently general and easily realized needed ingredients of the Zeldovich's model, such kind of the minimal liquid crystal has been never observed. However long after the publication of this work [7] the spontaneous chiral symmetry breaking has been observed and identified in the kingdom of the nematic liquid crystals [8-12]. Such a phase, termed as twist-bend nematic liquid crystal, $N_{T B}$, has short pitch $(\leq 10 \mathrm{~nm})$ heliconical structure with a doubly degenerate (left-right) spiral. The fact is especially surprising, because the conical spiral ordering is chiral, whereas the material is formed of non-chiral molecules. The $N_{T B}$ phase should be distinguished from the both known for ages liquid crystals: conventional nematics (also formed in achiral materials) with the uniform in the ground state orientational order characterized by the unit headless vector-director $\mathbf{n}$, and from the cholesteric liquid crystals formed in chiral materials, where the spiral axis is orthogonal to the director. Usually [8-12] the $N_{T B}$ phase is observed in materials formed by bent-shaped or dimer molecular building blocks. In such materials the $N_{T B}$ structure is observed upon cooling from a classical nematic liquid crystal $(N)$. Thus, the $N-N_{T B}$ transformation is the phase transition between two orientationally ordered phases, achiral $N$ and chiral $N_{T B}$.

Although the microscopical mechanisms leading to longrange nematic-like but heliconical (and therefore chiral) order in the $N_{T B}$ phase are to a large extent unknown, a number of phenomenological theoretical models have been proposed in the literature [13-22]. Aiming to contribute into the special issue of the Low Temperature Physics Journal devoted to I.M. Lifshitz (who was always very close to the Landau school of the theoretical physics), we develop and discuss in this work Landau phase transition theory for the $N_{T B}$ liquid crystals.

In a recent work [23] V.V. Lebedev with the author of this paper, have derived the Landau free energy functional 
describing the $N-N_{T B}$ transition. Since then (a few years after this work has been published) the new experiments $[24,25]$ show that for some compounds a direct transition between $N_{T B}$ liquid crystals and isotropic liquid ( $I$ ) takes place. Motivated by these new observations in what follows we generalize the Landau-like approach [23] to include the direct phase transition $I-N_{T B}$. In the next Sec. 2 we formulate the free energy model in terms of the tensor order parameter $Q_{i k}$. Then in the Sec. 3 we show that depending on the Landau expansion coefficients, our model allows either direct $I-N_{T B}$ transition, or the sequence of the phases $I-N-N_{T B}$ with the classical nematic liquid crystal sandwiched between the isotropic liquid and heliconical nematic liquid crystal. Which of these two situations is realized depends on how strong is the first order phase transition from the isotropic liquid. If it is strong enough the system undergoes $I-N-N_{T B}$ sequence, and for the very weak first order phase transition $I-N_{T B}$ transformation occurs. Furthermore in the latter case the $N_{T B}$ structure can be biaxial heliconical nematic liquid crystal. We summarize our main findings and shortly discuss further directions of investigations and perspectives in the conclusion Sec. 4.

\section{Landau model of the $N_{T B}$ phase}

In a spirit of the Landau theory approach to describe second order (or weak first order) phase transition, first of all one has to introduce the order parameter suitable for the phase transition under consideration. For the transition between $N$ and $N_{T B}$ liquid crystals, the natural choice of the order parameter is short-scale (since the heliconical spiral pitch is about $10 \mathrm{~nm}$ ) vector $(\phi)$ orthogonal to the nematic director $\mathbf{n}$. The minimal model, (including all relevant contributions and describing all essential features of the both phases, $N$ and $N_{T B}$, and phase transition between them) has been formulated in [23]. The Landau free energy expansion for this model reads as

$$
\begin{gathered}
\int d V\left\{\frac{a}{2} \phi^{2}+\frac{b_{3}}{8 q_{0}^{2}}\left[\left(n_{i} n_{k} \partial_{i} \partial_{k}+q_{0}^{2}\right) \phi\right]^{2}+\frac{b_{1}}{2}(\nabla \phi)^{2}+\right. \\
\left.+\frac{b_{\perp}}{2} \delta_{i j}^{\perp} \partial_{i} \phi \partial_{j} \phi+\frac{\lambda}{24} \phi^{4}-\frac{\lambda_{1}}{16 q_{0}^{2}}\left(\varepsilon_{i j k} \phi_{i} \partial_{j} \phi_{k}\right)^{2}\right\},
\end{gathered}
$$

where $\delta_{i j}^{\perp}=\delta_{i j}-n_{i} n_{j}$. As usual, $a \propto T-T_{c}$, where $T_{c}$ is the mean field transition temperature. All other coefficients in (1) are assumed to be temperature independent in the vicinity of the $N-N_{T B}$ transition temperature. The coefficients $b_{1}, b_{\perp}$, and $b_{3}$ are analogs of the Frank orientational elastic moduli for the order parameter $\phi$, and $q_{0}$ determines the pitch of the heliconical spiral. If $\lambda_{1}>0$, one can easily conclude, minimizing of the last term in (1) that for $T<T_{c}$

$$
\phi_{x}=A \cos \left(q_{0} z+\xi\right), \quad \phi_{y}= \pm A \sin \left(q_{0} z+\xi\right)
$$

where $A$ is the module of the order parameter $\phi, \xi$ is its phase and signs \pm correspond to clock-wise or anti-clockwise rotation of the conical spiral.

However the free energy expansion (1) assumes that the nematic director $\mathbf{n}$ is fixed along a specific direction and weakly fluctuates quantity, i.e., $\mathbf{n} \simeq \mathbf{n}_{0}=(0,0,1)$. Therefore it can not describe the direct $I-N_{T B}$ transition, where the order parameter is $3 \times 3$ symmetric traceless tensor $Q_{i k}$, [2], which is characterized by 5 independent parameters and not reduced to the two-component nematic director $\mathbf{n}$. Therefore to include into consideration the direct $I-N_{T B}$ transition, and to catch all observable features of the phase diagrams, the free energy expansion (1) should be generalized and rewritten in terms of the tensor order parameter.

The needed free energy functional (which allows to describe $I, N$, and $N_{T B}$ phases) contains the conventional nematic energy part

$$
\begin{aligned}
& F_{n}=\int d V\left\{\frac{a}{2} Q_{i k}^{2}-\frac{\mu}{6} Q_{i k}^{3}+\frac{\lambda}{24} Q_{i k}^{4}+\right. \\
& \left.+\frac{1}{2} L_{1}\left(\nabla_{j} Q_{i k}\right)^{2}+\frac{1}{2} L_{2}\left(\nabla_{i} Q_{i k}\right)^{2}\right\}
\end{aligned}
$$

where $a \propto\left(T-T_{*}\right)$ and other coefficients in (3) are temperature independent. Since (at $\mu \neq 0$ ) we are dealing with the first order phase transition, $T_{*}$ is the mean-field spinodal point (i.e., for $T<T_{*}$ the state with $Q_{i k}=0$ becomes unstable). The functional (3) leads to the equilibrium isotropic $I$ and classical nematic $N$ phases. To account for the $N_{T B}$ state, the functional (3) has to be supplemented by the non-uniform gradient terms favoring and stabilizing (in a certain range of parameters) the heliconical state $N_{T B}$. The terms (similar to those in (1)) read as

$$
F_{h c}=\int d V\left\{\frac{b_{3}}{8 q_{0}^{2}}\left[\left(\nabla^{2}+q_{0}^{2}\right) Q_{i k}\right]^{2}-\frac{\lambda_{1}}{16 q_{0}^{2}}\left(\varepsilon_{i j k} Q_{k l} \nabla_{j} Q_{i l}\right)^{2}\right\},
$$

where for the sake of brevity we use the same as in (1) notations for the coefficients, $\varepsilon_{i j k}$ is Levi-Civita $3 \mathrm{~d}$ order antisymmetric tensor. Since $q_{0}$ (inversely proportional to the heliconical spiral pitch) is not a small wave vector, one can add to the $F_{n}$ and $F_{h c}$ energies many other terms with the gradients $(\nabla \hat{Q})$, e.g., $\left(\nabla_{i} \nabla_{k} Q_{i k}\right)^{2},\left(\nabla_{n} \nabla_{m} Q_{i k}\right)^{2}$, and so on. The terms could change some symmetry features of the emerging $N_{T B}$ structure. Aiming in this work to describe only the very possibility to get the both experimentally observed sequences of phase transitions $\left(I-N_{T B}\right.$ and $\left.I-N-N_{T B}\right)$ and not interesting in detail symmetry of the $N_{T B}$ structure we neglect all higher order over $\nabla \hat{Q}$ terms.

With all said above in mind, the minimization of the free energy functional $F=F_{n}+F_{h c}$ is a simple straightforward procedure. We can present the order parameter $Q_{i k}$ as the sum of the uniaxial (classical nematic) part $Q_{i k}^{0}$, and the 
heliconical (fluctuational in the isotropic liquid and in the vicinity of the phase transition) part $\delta Q_{i k}$ :

$$
Q_{i k}=Q_{i k}^{0}+\delta Q_{i k},
$$

where

$$
Q_{i k}^{0}=s\left(n_{i} n_{k}-\frac{1}{3} \delta_{i k}\right)
$$

and

$$
\begin{gathered}
\delta Q_{i k}=\xi_{1}\left(n_{i} e_{k}+n_{k} e_{i}\right)+\xi_{2}\left(n_{i} g_{k}+n_{k} g_{i}\right)+ \\
+\xi_{3}\left(e_{i} g_{k}+g_{k} e_{i}\right)+\xi_{4}\left(e_{i} e_{k}-g_{i} g_{k}\right),
\end{gathered}
$$

where four functions $\xi_{i}(i=1, \ldots .4)$ determine the heliconical order parameter, and $\mathbf{e}$ and $\mathbf{g}$ are two unit vectors forming together with the classical director $\mathbf{n}$ the righthand triad. Note that if $\xi_{3,4} \neq 0$, the $N_{T B}$ structure has a biaxial symmetry, and for the vanishing values of the parameters $\xi_{3}=\xi_{4}=0$, the $N_{T B}$ structure has an uniaxial symmetry. Note to the point that this simple conclusion is in the agreement with recent numeric simulations of the twist-bend nematics [26].

\section{Mean field results}

Replacing in Eqs. (3), (4) the order parameter $Q_{i k}$ by its explicit form (5)-(7) we end up with the free energy functional in terms of the 5 parameters $s, \xi_{1}, \xi_{2}, \xi_{3}, \xi_{4}$ corresponding to the 5 independent components of the symmetric traceless tensor order parameter $Q_{i k}$. The results of the minimization crucially depends on the magnitudes of the coefficients $\mu$ and $b_{3} / q_{0}^{2}$. We omit the simple calculations and present only the results:

- If $\mu>b_{3} /\left(8 q_{0}^{2}\right)$, upon cooling from the isotropic liquid state, the first transition is the 1st order phase transition into the uniaxial classical nematic liquid crystal. Then decreasing further the temperature, the second order (in the mean-field approximation) between $N$ and the uniaxial $N_{T B}$ structure takes place.

- In the opposite case $\mu<b_{3} /\left(8 q_{0}^{2}\right)$ upon cooling from the isotropic fluid the first transformation occurs into the $N_{T B}$ structure. Depending on the higher order terms (neglected in (3), (4)), the $N_{T B}$ phase can acquire a certain degree of the biaxiality.

It is worth to noting that as it was shown almost 40 years ago [27], the smaller is the coefficient in front of the $3 \mathrm{~d}$ order over the order parameter term in the Landau free energy expansion ( $\mu$ in our notation), the smaller is the energy difference between the uniaxial and biaxial states. This conclusion is also confirmed by the numeric simulations [26].

\section{Conclusion}

In this paper we considered theoretically the possible phase diagrams for twist-bend nematic liquid crystals. In the framework of the Landau theory, including the least number of terms which allow to catch essential features of the short-scale heliconical structures, we found that there are possible two types of the equilibrium phase sequences:
$I-N-N_{T B}$ and $I-N_{T B}$. Moreover for the first sequence, the phase transition $I-N$ is more strong first order phase transition than the $I-N_{T B}$ transition in the second sequence. Besides for the latter case (with the small $3 \mathrm{~d}$ order over the order parameter term in the Landau free energy expansion) the biaxial $N_{T B}$ competes with the uniaxial heliconical nematic liquid crystal. Our results are in the qualitative agreement with experimental data [24,25] and numeric simulations [26].

Finally we note that the study of heliconical nematics is in its early stages. Many fundamental and applied challenges remain in the both, theory and experiment sides. To mention a few, for example, in our work we did not consider thermal fluctuational effects, which can be essential, especially for the almost continuous phase transitions $N-N_{T B}$ or $I-N_{T B}$. Such (and many related) theoretical investigations and systematic measurements would be highly desirable.

This work was funded by Russian Science Foundation (grant 14-12-00475). I acknowledge stimulating discussions with V.V. Lebedev who inspired this work.

1. S. Chandrasekhar, Liquid Crystals, Cambridge University Press, New York (1992).

2. P.G. de Gennes and J. Prost, The Physics of Liquid Crystals, Clarendon Press, Oxford (1993).

3. M. Kleman and O.D. Lavrentovich, Soft Matter Physics: An Introduction, Springer (2003).

4. S.A. Pikin, Structural Transformations in Liquid Crystals, Gordon and Breach, New York (1991).

5. L.M. Blinov and V.G. Chigrinov, Electrooptic Effects in Liquid Crystals, Springer, New York (1994).

6. P. Oswald and P. Pieranski, Nematic and Cholesteric Liquid Crystals: Concepts and Physical Properties Illustrated by Experiments, Liquid Crystals Book Series, Taylor and Francis, London (2005).

7. Ya.B. Zeldovich, ZhETF 67, 2357 (1971).

8. V.P. Panov, M. Nagaraj, and J.K. Vij, Phys. Rev. Lett. 105, 167801 (2010).

9. M. Cestari, S. Diez-Berart, D.A. Dunmur, A. Ferrarini, M.R. de la Fuente, D.J.B. Jackson, D.O. Lopez, G.R. Luckhurst, M.A. Perez-Jubindo, R.M. Richardson, J. Salud, B.A. Timimi, and H. Zimmermann, Phys. Rev. E 84, 031704 (2011).

10. V. Borshch, Y.-K. Kim, J. Xiang, M. Gao, A. Jakli, V.P. Panov, J.K. Vij, C.T. Imrie, M.G. Tamba, G.H. Mehl, and O.D. Lavrentovich, Nature Commun. 4, 2635 (2013).

11. D. Chen, J.H. Porada, J.B. Hooper, A. Klittnick, Y. Shen, M.R. Tuchband, E. Korblova, D. Bedrov, D.M. Walba, M.A. Glaser, J.E. Maclennan, and N.A. Clark, Proc. Natl. Acad. Sci. U.S.A. 110, 15931 (2013).

12. E. Gorecka, N. Vaupotic, A. Zep, D. Pociecha, J. Yoshioka, J. Yamamoto, and H. Takezoe, Angew. Chem., Int. Ed. 54 , 10155 (2015).

13. I. Dozov, Europhys. Lett. 56, 247 (2001).

14. V.L. Lorman and B. Mettout, Phys. Rev. E 69, 061710 (2004). 
15. E.G. Virga, Phys. Rev. E 89, 052502 (2014).

16. G. Barbero, L.R. Evangelista, M.P. Rosseto, R.S. Zola, and I. Lelidis, Phys. Rev. E 92, 030501 (2015).

17. C. Greco and A. Ferrarini, Phys. Rev. Lett. 115, 147801 (2015).

18. M.A. Osipov and G. Pajak, Eur. Phys. J. E 39, 45 (2016).

19. L. Longa and G. Pajak, Phys. Rev. E 93, 040701 (2016).

20. Nataša Vaupotič, Samo Curk, Mikhail A. Osipov, Mojca Čepič, Hideo Takezoe, and Ewa Gorecka, Phys. Rev. E 93, 022704 (2016).

21. A.G. Vanakaras and D.J. Photinos, Soft. Matter 12, 2208 (2016).
22. C. Meyer and I. Dozov, Soft. Matter 12, 574 (2016).

23. E.I. Kats and V.V. Lebedev, JETP Lett. 100, 110 (2014).

24. R.J. Mandle and J.W. Goodby, Soft Matter 12, 1436 (2016).

25. A.A. Dawood, M.C. Grossel, G.R. Luckhurst, R.M. Richardson, B.A. Timimi, N.J. Wells, and Y.Z. Yousif, Liq. Cryst. 43, 2 (2016).

26. W. Tomczyk, G. Pajak, and L. Longa, arxiv: 1606.0063v1 (2016).

27. V.L. Pokrovskii and E.I. Kats, Sov. Phys. JETP 46, 405 (1977). 